\title{
Development of a model of a homogeneous continuous medium based on the material with defects in the form of hollows
}

\author{
Vadim Poroshin ${ }^{1, *}$, Andrey Shlishevsky ${ }^{2}$, and Ksenia Tsybulya ${ }^{3}$ \\ ${ }^{1}$ Aerospace Dept., Polytechnic Institute, South Ural State University (National Research University), Chelyabinsk, Russian Federation \\ ${ }^{2}$ Aerospace Dept., South Ural State University (National Research University), Chelyabinsk, Russian Federation \\ ${ }^{3}$ Social-humanitarian Institute, South Ural State University (National Research University), Chelyabinsk, Russian Federation
}

\begin{abstract}
In a body of the billet received by casting, inevitably there are defects accompanying this process: blisters, hollows and other discontinuities affecting the deformation and strength properties of the metal. The experience showed that direct calculation of the stress-strain state by the FEM of the body with a defective structure requires the use of supercomputer-level computing power. A medium with multiple randomly distributed discontinuities was schematized by a regular structure formed by a set of elements in the form of a regular tetrahedron with spherical hollows at its vertices. The proposed technique makes it possible to create the model of a continuous homogeneous medium, which is equivalent in its deformation properties to the original discontinuous material. Using this approach was got a power-law approximation of the stress-strain curve of the model medium. The last one represents the basic characteristic of the material, both under singleshot loading and (if we take into account the cyclic hardening) in analyzing the kinetics of the stress and strain fields' under repeatedly-variable loading. The discontinuity of the material was fixed using the Kolmogorov's deformation criterion of ductile fracture. This criterion was applied in evaluating the limit state of the valve chamber under operating conditions.
\end{abstract}

Nowadays the pipeline transportation carries out the relocation of more than a half of all volume of hydrocarbons. An integral part of any pipeline is a different type of pipe fitting, through which realize the distribution of flows, the regulation of the outturn or the complete overlapping of the main. A significant part of the main pipeline fitting are valves having the impressive sizes and considerable weight (about several tons). In this regard, shells of similar products are received, as a rule, by casting, during which various defects arise in the billet: metallic and non-metallic inclusions, discontinuities, blisters. This circumstance negatively affects durability of the finished product, so much more that the endurance of such hardwares can be up to 50 years. It is needless to recall that the fracture of even a single main valve entails not only serious economic losses, but it also can threaten an ecological catastrophe.

Estimating the strength reliability of such constructions is usually based on two criteria: strength under a single-shot static loading (in particular, for control tests after manufacturing), and durability under repeatedly-variable loading under operating conditions. In both cases, it is necessary to calculate the stress-deformed state of the object, taking into account the inelastic behavior of the material under single and repeatedly-variable loading in combination with the corresponding criteria for achieving the limit state. Such an analysis you inevitably have to perform numerically, for example, by the finite element method (FEM) with the help of recognized computing power. At the same time, experience shows that direct calculation of the stress-strain state of the body with randomly distributed defects on its volume, even under single-shot, and, even more, under repeatedly-variable, loading, is very laborious process and requires the use of supercomputer-level computing power.

In connection with the foregoing, we propose the technique for determining the parameters of the model of a continuous homogeneous medium, which is equivalent in its deformation properties to the original material with multiple randomly distributed discontinuities.

Such an approach is not absolutely new, however in known for us works it was mainly used for ceramic classical brittle materials [1-3].

With regard to plastic materials, the problem arises in obtaining a stress-strain curve taking into account material inelasticity. To resolve it, the initial medium with defects was schematized with a regular structure formed by a set of elements in the form of a regular tetrahedron with spherical voids at its vertices (Fig. 1).

At the first stage we studied the effect of the parameters (the distance $h$ between the defects and eccentricity $e$ - displacements with respect to the medial surface of the valve chamber) of the structure single element (Fig.2) at a safety factor of the limit stress and the limit plastic strain. The diameter of the defect was assumed to be constant, equal to the maximum permissible for the most stressed zone of the shell in

\footnotetext{
Corresponding author: poroshin2816@mail.ru
} 
accordance with the regulatory documentation of manufacturer.

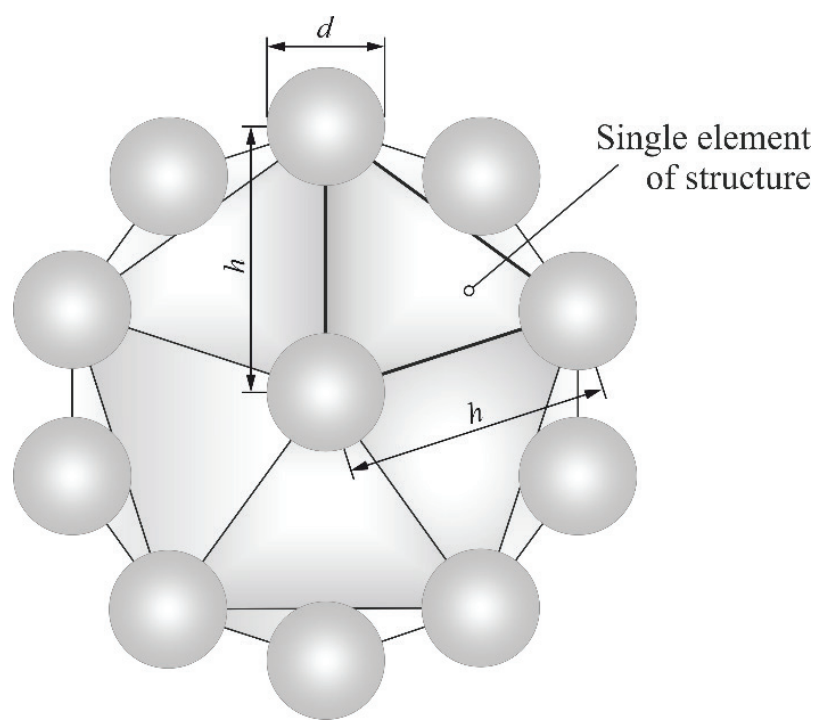

Fig. 1. The regular structure and its element - a tetrahedron.

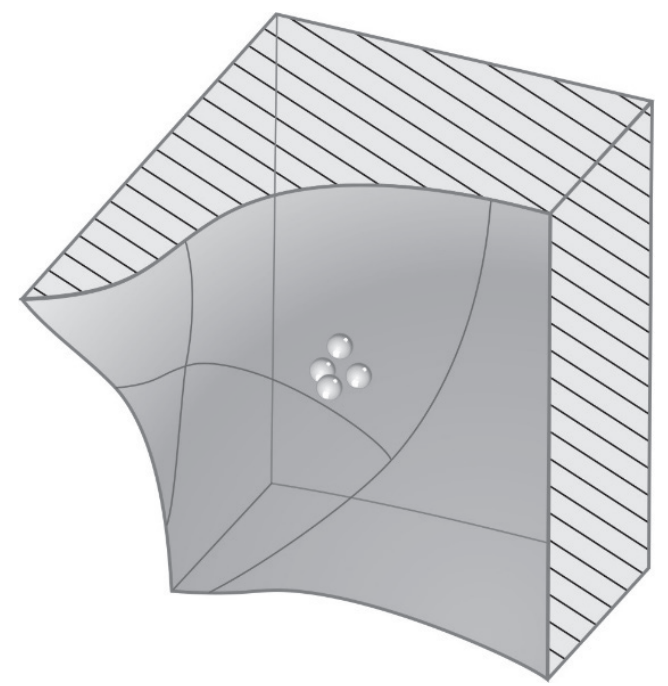

Fig. 2. A single element with defects in the shell wall.

Numerical analysis was performed by the finite element method using the ANSYS Workbench software. To increase the accuracy of calculation and reduce its labor intensity we used the submodeling procedure allocation of the zone which is needs to be analyzed and setting boundary conditions for this fragment by transferring obtained by calculation of the common «global» model of forces and displacements on the submodeling nodes. Such a technique is correct provided that the features of the allocated zone, in particular the stress concentrators, do not introduce a significant perturbation into the stress-strain state at the boundaries of the selected fragment. This method allows significantly reduce the total number of finite elements (FE) and use a more dense, refined mesh.

Thus, one of the most loaded regions of the valve chamber (the root region of the rib) was identified, where due to the relatively complex geometric shape there are high probability of existence of casting defects (Fig. 3).

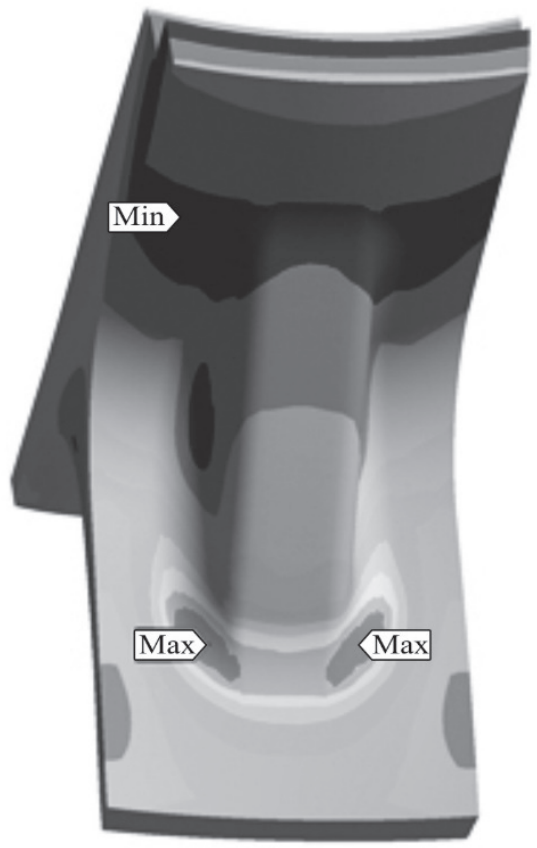

Fig. 3. The root part of the valve chamber ribs with the different size regions of equivalent von Mises stress under the working pressure ( $\sigma_{\text {eq }}^{\max }=255 \mathrm{MPa}$; inelastic calculation).

The use of a mesh of an irregular thickening hexahedral configuration made it possible to describe the stress and strain fields near spherical concentrators quite satisfactorily. The correctness of the FE object representation was confirmed by comparison with known solutions for linearly elastic material [4]. Fig. 4 shows the change of the theoretical concentration coefficient $k_{t}$ with increasing distance between two spherical defects. As they are retired, the coefficient $k_{t}$ tends to a value of 2.05, which is characteristic of a single concentrator; The error of the numerical solution does not exceed $2.5 \%$.

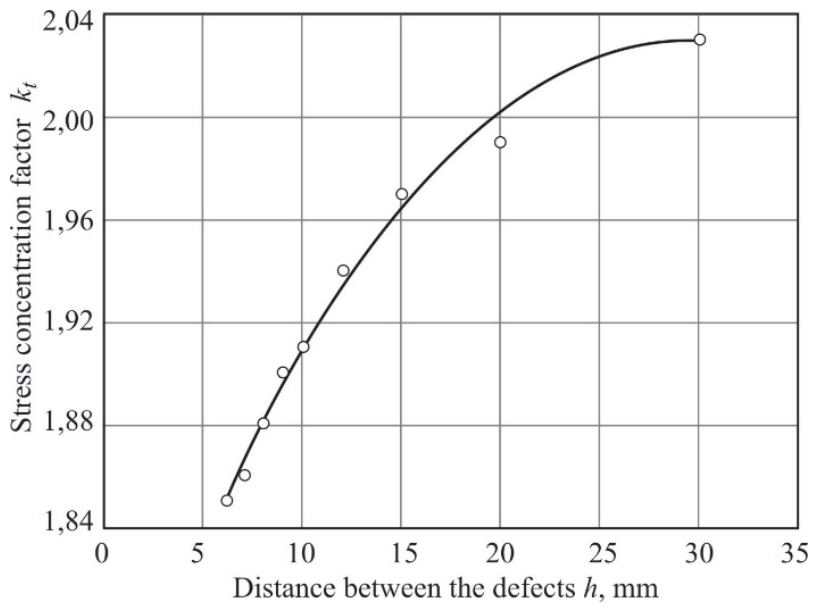

Fig. 4. The change of the theoretical concentration coefficient with increasing distance between two spherical defects.

In the same way, the finite element mesh was selected for four spherical concentrators located at the vertices of the tetrahedron. It is noted that when $h \approx d$, the greatest stresses occur at the places of tangency of discontinuities, in this case the concentration coefficient is equal to 5.57 . 
Taking into account that the value of the strain in the concentration region is usually significant, the strainstress curve of the steel was approximated by a power-law dependence by Ramberg-Osgood:

$$
\bar{\sigma}=K p^{m},
$$

where $\bar{\sigma}=\sigma(1+\varepsilon)=\sigma \exp (e)-$ true stress; $\sigma, \varepsilon-$ conditional stress and conditional (sometimes called as engineering) strain; $e=\ln (1+\varepsilon), p=\ln \left(1+\varepsilon_{p}\right)-$ total and plastic logarithmic strains; $K, m-$ constants of the material of the conditions under consideration.

During the study, the distance between the hollows was increased in $0.5 \mathrm{~mm}$ increments until the mutual influence of the stress fields around each concentrator became negligible. The variation of the eccentricity e of the defect location with respect to the median surface of the shell wall we made in both directions (towards the more loaded region - the internal surface of the wall - the value e was taken as positive, toward the less loaded region - the external wall surface - negative). With each combination of the parameters $h$ and $e$ we counted the values of the actual safety factor of the limit stress and strain,

$$
k_{p}=\frac{p_{\mathrm{int}}^{f}}{p_{\mathrm{int}}}, \quad k_{\sigma}=\frac{\sigma_{\mathrm{int}}^{f}}{\sigma_{\mathrm{int}}},
$$

corresponding to the current stress-strain state; $p_{\text {int }}^{f}=p_{\text {int }}^{f}\left(\frac{\sigma_{0}}{\sigma_{\text {int }}}\right)-$ limit (corresponding to the discontinuity) logarithmic plastic strain;

$$
\sigma_{0}=\frac{\sigma_{1}+\sigma_{2}+\sigma_{3}}{3}
$$

- mean stress;

$$
\sigma_{\text {int }}=\frac{1}{\sqrt{2}} \sqrt{\left(\sigma_{1}-\sigma_{2}\right)^{2}+\left(\sigma_{2}-\sigma_{3}\right)^{2}+\left(\sigma_{3}-\sigma_{1}\right)^{2}}
$$

- stress intensity;

$$
p_{\text {int }}=\frac{\sqrt{2}}{3} \sqrt{\left(p_{1}-p_{2}\right)^{2}+\left(p_{2}-p_{3}\right)^{2}+\left(p_{3}-p_{1}\right)^{2}}
$$

- logarithmic plastic strain intensity; $\sigma_{\mathrm{i}}$ - principal stresses; $p_{\mathrm{i}}-$ principal logarithmic plastic strains.

We determined the limit value $p_{\text {int }}^{f}$ with the aid of the deformation criterion of V. L. Kolmogorov [5], which represents the condition of ductile fracture under a complex stress state of general form under the conditions of a single-shot static load. This criterion can be written in two forms:

a) in terms of strain:

$$
\frac{p_{\text {int }}^{f}}{p_{f}}=a \exp \left(b \frac{\sigma_{0}}{\sigma_{\text {int }}}\right) ;
$$

here $p_{f}=\ln \left(\frac{1}{1-\psi}\right)$ - ductile resource under uniaxial tension ( $\psi$ - reduction of the area at fracture); $a, b-$ constants of the material of the conditions under consideration, which are determined in experiments on tension, compression, or pure shear;

b) taking into account the relationship between stress intensity and strain one by means of a generalized (general) stress-strain curve, in terms of stresses:

$$
\frac{\sigma_{\text {int }}^{f}}{\bar{\sigma}_{f}}=\left[a \exp \left(b \frac{\sigma_{0}}{\sigma_{\text {int }}}\right)\right]^{m} ;
$$

$\sigma_{\text {int }}^{f}-$ stress intensity at the moment of fracture; $\bar{\sigma}_{f}-$ ultimate tensile strength (actual stress at fracture).

Fig. 5 shows the field of the logarithmic plastic strain intensity in the boundary layer of the group defect, which corresponds to the internal pressure in the hollow of the valve under operating conditions. The maximum value of $p_{\mathrm{int}}^{\max }=0,32 \%$, while the intensity of the logarithmic plastic strain at the moment of fracture corresponding to this stress state $p_{\text {int }}^{f}=63.7 \%$; the actual safety factor for the limit strain $k_{p} \cong 200-$ it would seem that the value is more than sufficient, but in this case the limit stress safety factor $k_{\sigma}$ is only 1.38 !

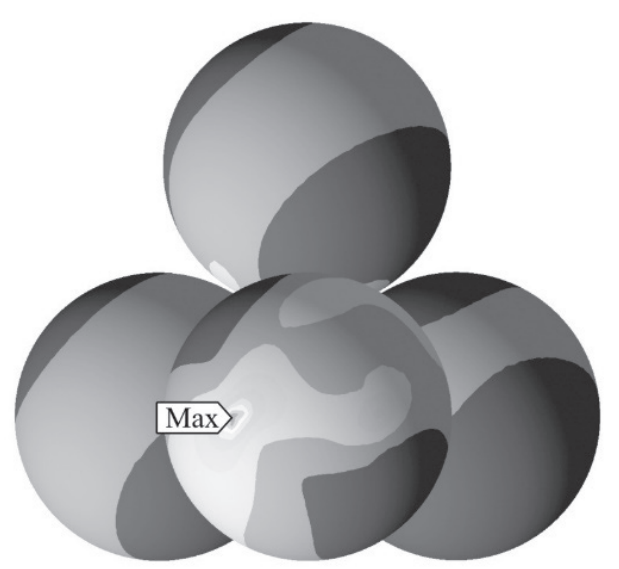

Fig. 5. Distribution of the intensity of logarithmic plastic strain in the boundary layer of the group defect under the working pressure (distance $h \approx 6 \mathrm{~mm}$; eccentricity $e=0 ; p_{\text {int }}^{\max }=0,32$

$\%)$.

Such an essentially nonlinear connection between the parameters «stress intensity» «strain intensity» is determined by a slight hardening of the steel $(m=0.06)$ in the process of inelastic deformation.

It is worth noting that the given value of the safety factor $k_{\sigma}$ is calculated by the limit stress $\sigma_{\text {int }}^{f}$. If to determine with respect to the ultimate tensile stress $\sigma_{\mathrm{B}}$ then this value will decrease to $k_{\sigma}=1.12$. In the case of estimating by the yield point $\sigma_{0.2}$, as is customary in engineering practice, the safety factor is less than unity $k_{\sigma}=0.96$, as it was expected in the presence of stress concentration. 

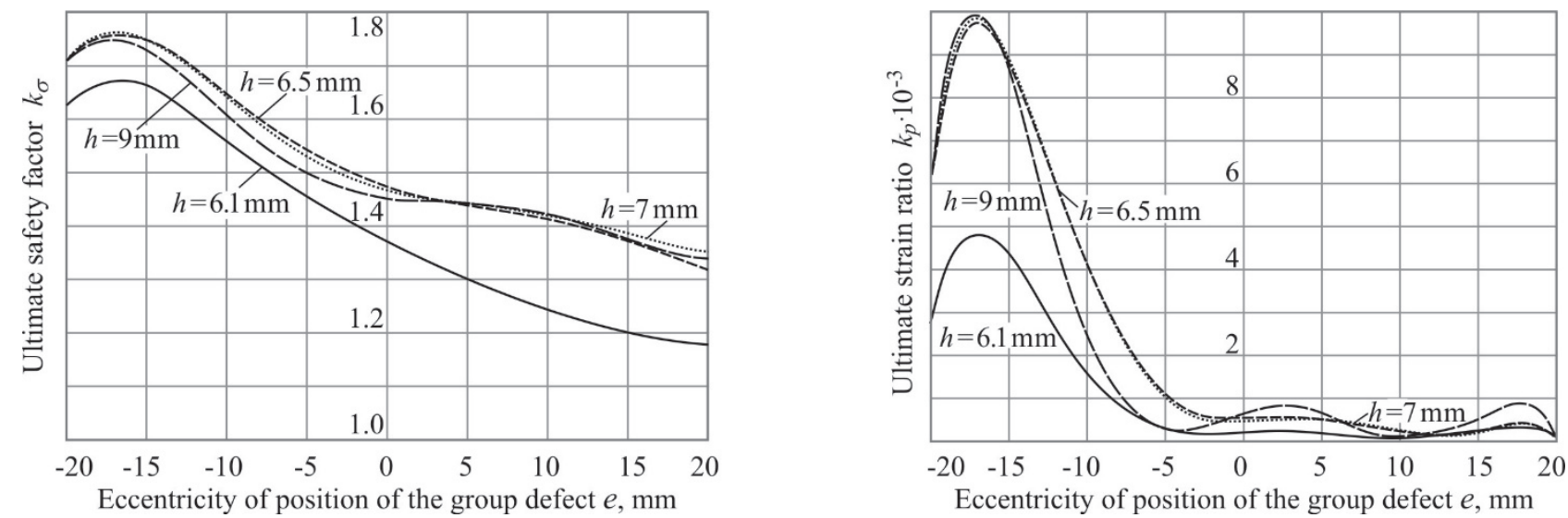

Fig. 6. Dependences of the safety factors by the limit strain and stress in connection with the distance between the discontinuities and the eccentricity of the arrangement of the group defect.

The correlation dependences of the safety factors by the limit strain and stress in connection with the distance between the discontinuities and the eccentricity of the arrangement of the group defect are illustrated by graphs (Fig. 6).

The evaluation of the durability of products under repeatedly-variable loading conditions by the criterion of fatigue fracture is made on the basis of a model of a continuous homogeneous elastoplastic medium, which makes it possible to use verified by engineering practice criterion relationships and the acceptable labor intensity methods of calculating. In this connection, the problem arises of modeling a medium with the indicated properties equivalent in its deformation characteristics to the original material with multiple randomly distributed discontinuities.

The equivalence criteria are the two conditions called by the authors «an integral stress»

$$
\sigma_{\Sigma}=\frac{1}{V_{0}} \int_{V} \sigma_{\text {int }} d V
$$

and «an integral deformability»

$$
\varepsilon_{\Sigma}=\frac{1}{V_{0}} \int_{V}\left|e_{\mathrm{i}}\right| d V
$$

or

$$
p_{\Sigma}=\frac{1}{V_{0}} \int_{V}\left|p_{\mathrm{i}}\right| d V
$$

- in the case of calculations in the parameters «true stress» $\sim$ «logarithmic plastic strain»; index «ii», as before, means belonging to principal stresses and strains.

Using this approach, was solved the problem of identifying the model - obtaining a stress-stain curve depending on the porosity of the material - based on the results of at least four (in the case of approximating the diagram by the Ramberg-Osgood power-low function) numerical experiments (Fig.7). Two of them are produced under the assumption of linearly elastic behavior of the material, the rest of them are elastoplastic. By the degree of porosity is meant the ratio of the volume of spherical hollows to the total volume of the substructure.

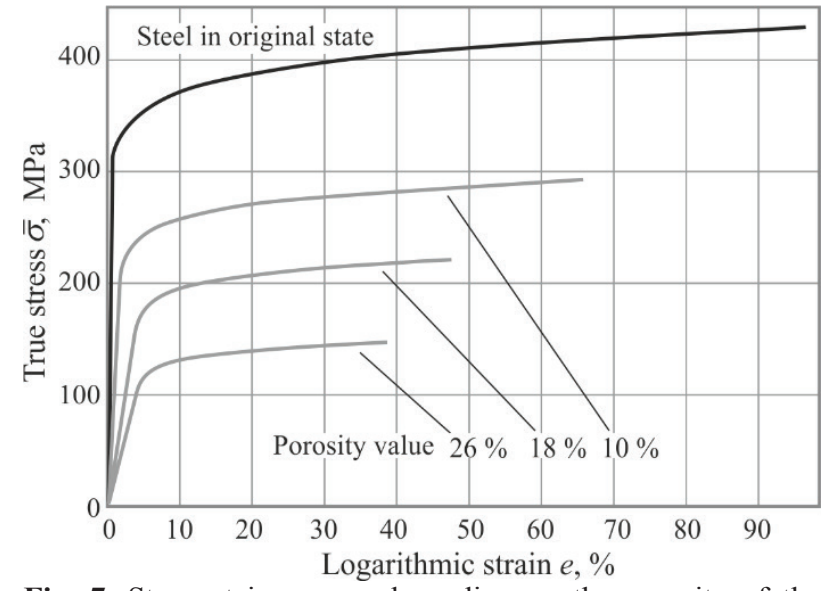

Fig. 7. Stress-stain curves depending on the porosity of the material.

In our opinion, further development of this work is in the comprehensive experimental verification of the proposed approach and improvement of criteria that establish the equivalence (in the said meaning) of a continuous model and real porous medium on this basis. In addition, it is proposed to develop a method of reckoning the stress concentration in the neighborhood of an actual defect, but absent in the model medium.

\section{References}

1. J.D. Clayton, R.H. Kraft, R.B. Leavy, Int. Journal of Solids and Structures, 49, pp. 2686-2702 (2012)

2. A.I. Slutsker, A.B Sinani, S.S. Ordanyan, V.I. Betekhtin, A.A. Kozhushko, A.G. Kadomtsev, Phys. of the Solid State, 50, no.8, pp. 1450-1457 (2008)

3. A.V. Ignatova, O.A. Kudryavtsev, S.B Sapozhnikov, PNRPU Mechanics Bulletin 4, pp. 121-137 (2015)

4. R.E. Peterson, Stress concentration factors (John Wiley and Sons, Inc., 1974)

5. V.L. Kolmogrov, Mechanics of metal forming (in Russion) (Publishing house of Ural state technical University, 2001) 